Ann. Biol. anim. Bioch. Biophys., I968, 8 (I), 95-98.

\title{
HETEROGENEITÉ DES PROPORTIONS MENDÉLIENNES A PLUSIEURS LOCI CHEZ LA POULE, LORSQUE LA MÈRE EST HÉTEROZYGOTE
}

\author{
P. MÉRAT \\ Station centrale de Génétique animale, \\ Centre national de Recherches zootechniques, 78 - Jouy-en-Josas \\ Institut national de la Recherche agronomique
}

Lors d'accouplements du type $\sigma^{*} r r$ (crête simple) $\times$ q $\mathrm{R} r$ (crête en rose), comportant au total environ 15000 descendants, nous avions observé une hétérogénéité entre mères, significative au seuil I p. roo, pour la proportion des crêtes simples par rapport aux crêtes en rose chez les descendants, sexes groupés; cette proportion était voisine de I/I sur l'ensemble des données (MÉRAT, I963 $a, b ;$ I966 a).

La fertilité et l'éclosion étaient normales dans ces familles, et ne présentaient pas de corrélation décelable avec le rapport de ségrégation pour le type de crête. D'autre part, la comparaison des proportions mendéliennes pour les divers types de croisement relativement au gène $R$ ne s'accordait pas avec l'hypothèse de gènes modificateurs présents dans certaines familles (MÁRAT, I963 a).

De même, le croisement $\sigma^{*}$ rw (pattes jaunes) $\times$ o Ww (pattes blanches) comporte une hétérogénéité entre mères, hautement significative, du rapport de ségrégation. Le pourcentage de poussins éclos rapporté aux œufs incubés ne diffère pas entre les mères à excès ou défaut significatif d'enfants à pattes blanches, et les autres mères accouplées aux mêmes pères. Ce résultat s'accorde mal avec l'hypothèse de différences dans la mortalité zygotique. Il ne s'agit pas non plus de désaccords entre le phénotype noté et le génotype ; les cas où un animal ww est noté "à pattes blanches " sont très rares et non groupés par familles. Il en est de même, en sens inverse, pour les animaux WW ou Ww (MÉRAT, $1966 b$ ).

Pour la même population, nous avons constaté (MÉRAT, I966a) des faits analogues au locus $\mathrm{C} / c$ (présence/absence de pigmentation du plumage). Dans nos croisements pedigree $\sigma^{t} c c \times$ o $C c$, le $\chi^{2} \mathrm{~d}^{\prime}$ hétérogénéité entre mères intra-pères, pour la proportion, sur les deux sexes réunis, de poussins colorés et blancs, est égal à 32I, 9 pour 265 degrés de liberté, valeur ayant une probabilité inférieure à I p. Ioo d'être dépassée du seul fait du hasard. 
Le tableau suivant (tabl. I) montre le taux d'éclosion chez les mères à excès significatif de poussins blancs (seuil $5 \mathrm{p}$. Ioo) comparé à celui des autres mères accouplées aux mêmes coqs (comparaison I) ; de même, le taux d'éclosion chez les mères présentant un défaut significatif de poussins blancs, comparé à celui des autres mères (comparaison II). Les différences de pourcentages ne sont pas significatives.

\section{TABLEAU I}

Taux d'éclosion des mères $\mathrm{C} c$, en relation avec leur pourcentage d'enfants colorés et blancs

\begin{tabular}{|c|c|c|c|c|}
\hline & \multicolumn{2}{|c|}{$\begin{array}{l}\text { Mères à excès (ou défaut) } \\
\text { de poussins blancs }\end{array}$} & \multicolumn{2}{|c|}{$\begin{array}{l}\text { Autres mères accouplées } \\
\text { aux mêmes pères }\end{array}$} \\
\hline & $\begin{array}{l}\text { Nbre d'œufs } \\
\text { incubés }\end{array}$ & $\begin{array}{l}\text { Pour cent } \\
\text { éclos }\end{array}$ & $\begin{array}{l}\text { Nbre d'œufs } \\
\text { incubés }\end{array}$ & $\begin{array}{l}\text { Pour cent } \\
\text { éclos }\end{array}$ \\
\hline $\begin{array}{c}\text { Comparaison I } \\
\text { (excès) }\end{array}$ & 228 & 74,13 & 1232 & 75,24 \\
\hline$\underset{\text { (défaut) }}{\text { Comparaison II }}$ & 104 & 78,84 & 410 & 77,32 \\
\hline
\end{tabular}

Dans notre cheptel, 1a présence ou l'absence de coloration du plumage ne présentait pas de difficulté de classification, d'autant plus que la population analysée pour la ségrégation $\mathrm{C} / c$ était fixée à l'état homozygote pour l'allèle $s$ (présence de mélanines " rouges" dans le plumage). En outre, les gènes I (" blanc dominant "), $\mathrm{Bl}$ (dilution du noir) et $i g$ (suppresseur de la pigmentation rouge) étaient absents des familles étudiées.

On ne voit donc pas la possibilité d'expliquer l'hétérogénéité observée par la présence de gènes suppresseurs de la pigmentation autres que $c$. Il y a d'ailleurs aussi souvent excès que défaut de poussins colorés.

On peut noter que les croisements réciproques, aux trois loci dont nous venons de faire mention ( $\sigma^{*} R r \times q r r ; \delta^{\alpha} W r \times q$ rw ; $\left.\sigma^{\star} C c \times q c c\right)$, ne présentent pas eux, d'hétérogénéité significative des proportions suivant les familles de mères. Entre pères, cette hétérogénéité est à la limite de la signification au locus $\mathrm{W}$, $(\mathrm{P} \simeq 0,05)$ ainsi qu'au locus $\mathrm{C}(P<0, \mathrm{ro})$ et au locus $R$ pour la proportion chez les mâles $(\mathrm{P} \simeq 0,05)$. (MÉRAT, I962, I966 $a$, I966 b).

Dans l'ensemble, les hétérogénéités de proportions constatées paraissent provenir surtout de quelques mères ayant un fort excès ou défaut d'une catégorie. Ceci est suggéré par l'examen de la distribution des $\chi^{2}$ calculés sur la descendance de chaque mère (proportion observée comparée à la proportion théorique $\mathrm{I} / \mathrm{I}$ ).

Chacune de ces quantités calculées suit approximativement, dans 1'hypothèse d'homogénéité, une loi du $\chi^{2}$ à I degré de liberté. Nous avons découpé la distribution théorique de cette loi en classes, avec les limites suivantes :

$$
0 ; 0,0158 ; 0,0642 ; 0,148 ; 0,455 ; 1,074 ; 1,642 ; 2,706 ; 3,841
$$

(la dernière classe comprenant toutes les valeurs supérieures à $3,84 \mathrm{I}$ ). 
On peut, à partir de là, comparer les nombres observés des $\chi^{2}$ obtenus pour les différentes mères aux nombres théoriques, dans chacune des classes formées, par un $\chi^{2}$ d'ajustement à 8 degrés de liberté (nombre de classes moins un).

Ce test indique que, pour le locus $R$, la distribution des $\chi^{2}$ observés s'écarte considérablement de la distribution théorique du $\chi^{2}$ (probabilité inférieure à $0,00 \tau$ ). pour C.

La probabilité correspondante est inférieure à 0,02 pour le locus $W$ et à 0,05

Pour le locus $\mathrm{R}$, en particulier, la comparaison des nombres observés et calculés de $~ \chi^{2}$ de mères individuelles » dans les diverses classes est donnée dans le tableau 2.

\section{TABLEAU 2}

Distribution observée, par classes, des $\chi^{2}$ par mère vis-à-vis de la proportion $1 / 1$, comparée a la distribution théorique

\begin{tabular}{|c|c|c|c|c|c|c|c|c|c|}
\hline & \multicolumn{9}{|c|}{ Valeurs du $\chi^{2}$ par classes } \\
\hline & $\begin{array}{c}0,0 \\
\stackrel{a}{\mathbf{a}} \\
0,0158\end{array}$ & $\begin{array}{c}0,0158 \\
\stackrel{a}{ } \\
0,0642\end{array}$ & $\begin{array}{c}0,0642 \\
\grave{a} \\
0,148\end{array}$ & $\begin{array}{c}0,148 \\
\mathrm{a} \\
0,455\end{array}$ & $\begin{array}{c}0,455 \\
\grave{a} \\
1,074\end{array}$ & $\begin{array}{c}1,074 \\
\mathbf{a} \\
1,642\end{array}$ & $\mid \begin{array}{c}1,642 \\
\mathrm{a} \\
2,706\end{array}$ & $\begin{array}{c}2,706 \\
\grave{a} \\
3,841\end{array}$ & $\underset{3,841}{>}$ \\
\hline $\begin{array}{l}\text { Nombres théoriques } \\
\text { Nombres observés }\end{array}$ & $\begin{array}{l}66,4 \\
81\end{array}$ & $\begin{array}{l}66,4 \\
53\end{array}$ & $\begin{array}{l}66,4 \\
69\end{array}$ & $\begin{array}{l}132,8 \\
119\end{array}$ & $\begin{array}{l}132,8 \\
127\end{array}$ & $\begin{array}{l}66,4 \\
51\end{array}$ & $\begin{array}{l}66,4 \\
75\end{array}$ & $\begin{array}{l}33,2 \\
38\end{array}$ & $\begin{array}{l}33,2 \\
51\end{array}$ \\
\hline
\end{tabular}

Si l'on compare les nombres observés et théoriques des $\chi^{2}$ respectivement supérieurs et inférieurs à la valeur 3,84 , on obtient un $\chi^{2}$ de contingence égal à 24,5 pour I degré de liberté, d'où $\mathrm{P}<0,00 I$. L'ensemble des écarts dans les autres classes ne paraît pas excéder les fluctuations aléatoires normalement prévisibles.

Enfin, sur une souche Wyandotte et une autre de Rhode-Island, la ségrégation de deux loci de groupe sanguin dans chacune, fait apparaître une hétérogénéité semblable des proportions entre familles de mères lorsque la mère est hétérozygote et le père homozygote ; là encore, des différences de mortalité embryonnaire ou juvénile variables suivant la famille paraissent pouvoir être exclues (PERRAMON et MÉRAT, sous presse).

Dans ces divers cas, en l'absence d'une autre explication satisfaisante, nous sommes amenés à supposer que certaines variations non aléatoires du rapport de ségrégation pourraient se produire lors de la méiose ou avant elle, au moins chez certaines mères.

Plusieurs hypothèses, entre lesquelles nous n'avons pas actuellement le moyen de choisir, sont possibles. En dehors d'une anomalie de ségrégation à la méiose (expulsion préférentielle d'un allèle dans le globule polaire, variable suivant les familles) l'existence de " conversions géniques " pourrait être suggérée. Ce phénomène a été rencontré dans diverses espèces, avec peut-être des fréquences d'apparition importantes dans certains cas (par exemple RENNER, I942 ; CoE, I959 ; PAPAZIAN et LINDEGREN, I960). 
Il existe encore une autre possibilité, celle de crossing-over mitotiques dans la lignée germinale femelle ( $\left.{ }^{1}\right)$ montrée chez les Champignons et les Diptères (STERN, I936 ; Ponteconvo, I956, I958 entre autres). Récemment, cette possibilité a été fortement suggérée chez les Vertébrés supérieurs par GRÜNEBERG (Ig66) et par BATEMAN (I967).

La répétition d'un phénomène comparable pour divers loci chez la poule peut en faire un matériel intéressant pour tester ces diverses possibilités.

\section{Reşu pour publication en novembre 1967.}

\section{SUMMARY}

\section{HETEROGENEITY OF MENDELIAN PROPORTIONS AT SEVERAL LOCI IN THE DOMESTIC FOWL WITH AN HETEROZYGOUS DAM}

Matings of the $o c c \times$ i $\mathrm{C} c$ type corresponding to the $\mathrm{C} / c$ locus of the domestic fowl (pigmented. v. white plumage) were carried out in our population.

There was a highly significant heterogeneity between dams in the segregation ratio of their progeny, with both sexes pooled. This fact cannot be attributed to an incomplete penetrance of either allele ; and differences in zygotic mortality are as untenable an hypothesis.

The results of our experiments have led us to suggest a disturbance of the proportions at the gametic level. We obtained similar results with the $\mathrm{R}$ and $\mathrm{W}$ loci, and with blood group genes.

\section{RÉFÉRENCES BIBLIOGRAPHIQUES}

Bateman A. J., 1967. A probable case of mitotic crossing-over in the mouse. Genet. Res , 9. 375-376.

COE E. H. Jr., I 959. A regular and continuing conversion-type phenomenon at the B locus in maize. Proc. Nat. Acnd. Sci. U. S. 45. 828-8.32.

GrǘnEBERG H., I966. The case for somatic crossing-over in the mouse. Genet. Res., 7, 58-75.

MÉrat P., 1962. Ségrégations anormales pour les allèles "crête simple " et "crête en rose " chez la poule. I. Croisement ô $\mathrm{R} r \times$ of $r r$. Ann. Biol. anim. Biochim. Biophys., 2, 109-11 7 .

MERAT P., I963a. Abnormal segregation for the alleles "single Comb " and "rose comb " in the domestic fowl. Proc. Xlih Cong. Genel., I, 259 (abstr.).

MÉRAT P., I 963 b. Ségrégations anormales pour les allèles "crête simple " et "crête en rose " chez la poule. II. Croisement of $r r \times \$ \mathrm{Rr}$. Ann. Biol. anim. Biochim. Biophys., 3, 59-64.

Mérat P., I966 a. Contribution d̀ l'étude de la "valeur sélective " associée à quelques gènes chez la poule domestique. Thèse, Paris.

Mérat P., I966 b. Irrégularités des proportions mendéliennes au locus W/w chez la poule domestique. Ann. Biol. anim. Biochim. Biophys., 6, 249-254.

Papazian H. P., Lindegren $\quad$ C. C., I960. A study of irregular quadruplets in Saccharomyces. Genelics, 45, 847-854.

Perrayon A., MÉrat P., I967. Étude génétique des groupes sanguins dans deux populations de volailles. I. Transmission héréditaire des facteurs antigéniques. Ann. Biol. anim. Biochim. Biophys. (Sous presse).

Ponteconvo G., 1956. The parasexual cycle in fungi. Ann. Rev. Microbiol., 10, 393-400.

Pontecorvo 1958. Trends in genetic analysis. X + $145 \mathrm{pp.} \mathrm{Columbia} \mathrm{University} \mathrm{Press,} \mathrm{New} \mathrm{York.}$

Proust J., I964. Étude de l'action génétique de la lumière ultravioletie sur les ovogonies de drosophiles Thèse, Paris.

RENNER O., 1942. Uber den Erbgang des cruciata-merkmals der Enotheren. III. Weitere Belge für somatische Konversionen. Z. Vererb. Lehre, 80, 570-589.

STERN C., I936. Somatic crossing-over and segregation in drosophila melanogaster. Genetics, 21, 625-730.

(1) Ou tout autre mécanisme conduisant à un mosaïcisme des gonades. 